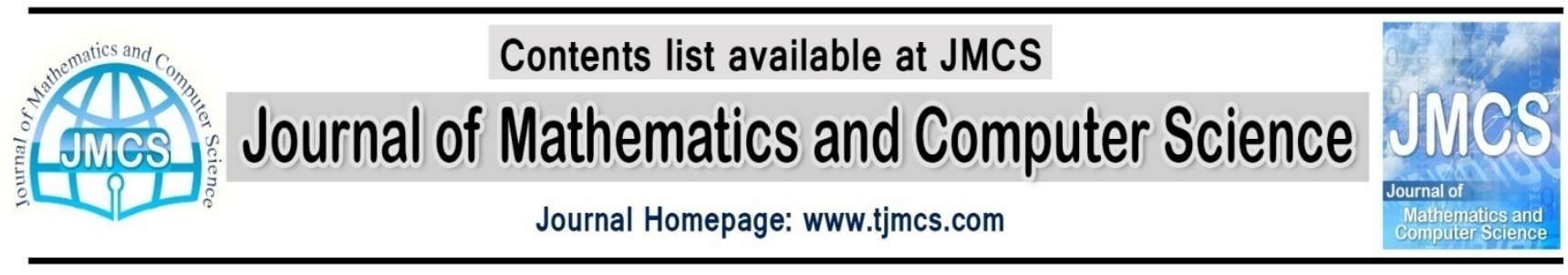

\title{
Sumudu transform method for solving fractional differential equations and fractional Diffusion-Wave equation
}

\author{
Rahmat Darzi \\ Department of Mathematics, Islamic Azad University Neka Branch, Neka, Iran \\ r.darzi@iauneka.ac.ir \\ Bahar Mohammadzade \\ Department of Mathematics, Islamic Azad University Sari Branch, Sari, Iran \\ bahar@math.com \\ Sahar Mousavi \\ Department of Mathematics, University of Mazandaran Babolsar, Iran \\ mousavi.math@yahoo.com \\ Rozita Beheshti \\ Department of Mathematics, University of Mazandaran Babolsar, Iran \\ Beheshti@umz.ac.ir
}

Article history:

Received August 2012

Accepted November 2012

Available online January 2013

\begin{abstract}
In this paper, we obtain the solutions of a cauchy problems for differential equations with the Caputo fractional derivative and the solution of fractional Diffusion-Wave equation by using Sumudu transform techniques. The results presented here are in compact and elegant expressed in term of Mittag-Leffler function and generalized Mittag-Leffler function which are suitable for numerical computation.

Keywords: Sumudu transform, Fractional differential equation, Diffusion-Wave equation.
\end{abstract}

2010 Mathematics Subject Classification: Primary 26A33; Secondary 44A35.

\section{Introduction}

In turns out that the Sumudu transform has very special and useful properties and can help with intricate applications in science and engineering. The sumudu transform, was proposed originally by Watugala [1,2,3]. In [4,5] some fundamental properties of the Sumudu transform were established. Subsequently exploited by Weerakoon $[6,7]$ and they have been used by Chaurasia et 
al. for Schodinger equation [8]. Recently Kilicman et al. [9] applied this transform to solve the system of differential equations.

In this work, we apply Sumudu transform method to derive fundamental system of solutions to homogeneous equation of the following form

$$
D_{0^{+}}^{\alpha} y(t)-\lambda y(t)=0, \quad \lambda \in \mathbb{R}, \quad m-1<\alpha \leq m, \quad m \in \mathbb{N}
$$

subject to

$$
y^{(k)}(0)=b_{k}, \quad k=0, \ldots, m-1,
$$

and the solutions of fractional Diffusion-Wave equation

$$
\frac{\partial^{\alpha} U(x, t)}{\partial t^{\alpha}}=c^{2} \frac{\partial^{\beta} U(x, t)}{\partial x^{\alpha}}
$$

with the boundary conditions

$$
U(x, 0)=f_{1}(t), \quad \frac{\partial}{\partial t} U(x, 0)=f_{2}(t)
$$

where $f_{i}(t), i=1,2$ are continuous and $c$ is constant.

Definition1. ([1,2]) The Sumudu transform over the set functions

$A=\left\{f(t)\left|\exists M, \tau_{1}, \tau_{2},\right| f(t) \mid<M e^{\frac{|t|}{\tau_{j}}}\right.$, if $\left.t \in(-1)^{j} \times[0,+\infty)\right\}$,

is defined by

$$
G(u)=S[f(t)]=\int_{0}^{\infty} f(u t) e^{-t} d t, \quad u \in\left(-\tau_{1}, \tau_{2}\right),
$$

Definition 2. ([10,11]) A real function $f(t)>0, t>0$ is said to be in the space $C_{\mu}, \mu \in \mathrm{R}$, if there exists a real number $n(>\mu)$, such that $f(t)=t^{n} f_{1}(t)$,, where $f_{1}(t) \in C[0, \infty)$, and it is said to be in the space $C_{\mu}^{k}$ if and only if if and only if $f^{(k)} \in C_{\mu}, k \in \mathbb{N}$.

Definition 3. ([10,11]) The Riemann-Liouville fractional integral operator of order of $\alpha>0$, of a function $f \in C_{\mu}, \mu \geq-1$ is defined as

$$
\begin{gathered}
I_{a}^{\alpha} f(t)=\frac{1}{\Gamma(\alpha)} \int_{a}^{t}(t-s)^{\alpha-1} f(r) d r, \\
I^{\alpha} f(t)=I_{0^{+}}^{\alpha} f(t) .
\end{gathered}
$$

Definition 4. ([10,13]) The fractional Caputo's derivative of $f$ is given by

$$
D^{\alpha} f(t)=I^{k-v} D^{k} f(t)=\frac{1}{\Gamma(k-\alpha)} \int_{0}^{t}(t-r)^{k-\alpha-1} f^{(k)}(r) d r
$$


where, $f \in C_{-1}^{k}, \quad k-1<\alpha \leq k, \quad k \in \mathbb{N}$.

Definition 5. ([10,11]) The Mittag-Leffler function $E_{\alpha}(z)$ for $\alpha>0$ and $z \in \mathbb{C}$ is defined by the series representation

$$
E_{\alpha}(z)=\sum_{k=0}^{\infty} \frac{z^{k}}{\Gamma(k \alpha+1)}, \quad \alpha>0, \quad z \in \mathbb{C}
$$

and generalized Mittag-Leffler function $E_{\alpha, \beta}(z)$ is defined by

$$
\begin{aligned}
& E_{\alpha, \beta}(z)=\sum_{k=0}^{\infty} \frac{z^{k}}{\Gamma(k \alpha+\beta)}, \quad \alpha, \beta>0, \quad z \in \mathbb{C} . \\
& E_{\alpha, \beta}^{(\delta)}(z)=\sum_{k=0}^{\infty} \frac{(\delta)_{k} z^{k}}{\Gamma(k \alpha+\beta) k !}, \quad \alpha, \beta>0, \quad \delta, z \in \mathbb{C} .
\end{aligned}
$$

where $(\delta)_{0}=1$ and for $k \in \mathbb{N}:(\delta)_{k}=\delta(\delta+1) \ldots(\delta+k-1)$.

Definition 6. ([12]) Linearly independent solutions of the equation (1) form the fundamental system of solutions if

$$
\left\{\begin{array}{c}
y_{j}^{(k)}(0)=0, \quad k, j=0, \ldots, m-1, \quad k \neq j \\
y_{j}^{(k)}(0)=1, \quad k=0, \ldots, m-1, \quad k=j
\end{array}\right.
$$

\section{Main Results.}

Lemma 2.1. The following results are satisfied to inverse Sumudu transform

$$
\begin{aligned}
& \text { i. } S^{-1}\left[u^{\gamma-1}\left(1-\omega u^{\beta}\right)^{-\delta}\right]=t^{\gamma-1} E_{\beta, \gamma}^{\delta}\left(\omega t^{\beta}\right) \\
& \text { ii. } \left.S^{-1}\left[\frac{u^{-\rho}}{u^{-\alpha}+a u^{-\beta}+b}\right]\right]=\sum_{r=0}^{\infty}(-a)^{r} t^{(\alpha-\beta) r+\alpha-\rho} E_{\alpha, \alpha+(\alpha-\beta) r-\rho+1, \gamma}^{r+1}\left(-b t^{\beta}\right) .
\end{aligned}
$$

Proof. $\boldsymbol{i}$.It can be easily established by expanding the binomial function

appearing in $i$ and interpreting it with the help of formula $S^{-1}\left[u^{n} ; t\right]=\frac{t^{n}}{\Gamma(n+1)}$. [8]

ii. To find inverse Sumudu transform of this function, we have

$$
\frac{u^{-\rho}}{u^{-\alpha}+a u^{-\beta}+b}=\frac{u^{-\rho+\beta}}{\left(u^{-\alpha+\beta}+b u^{\beta}\right)\left(1+\frac{a}{u^{-\alpha}+b u^{\beta}}\right)}=\sum_{r=0}^{\infty}(-a)^{r} u^{(\alpha-\beta) r+\alpha-\rho}\left(1-b u^{\alpha}\right)^{-r-1} .
$$

Now, using result $\$ \mathrm{i} \$$, it gives the desired result.

Definition 7. ([8]) The Sumudu transform of the Caputo's derivative of the function $f$ is given by 


$$
\begin{array}{cc}
S\left[D_{t}^{\alpha} f(t) ; u\right]=u^{-\alpha} \bar{f}(u)-\sum_{r=0}^{m-1} \frac{f^{(k)}(0)}{u^{\alpha-k}}, & m-1<\alpha \leq m . \\
S\left[D_{t}^{\alpha} f(x, t) ; u\right]=u^{-\alpha} \bar{f}(x, u)-\sum_{r=0}^{m-1} \frac{f^{(k)}(x, 0)}{u^{\alpha-k}}, & m-1<\alpha \leq m .
\end{array}
$$

Now, we apply Sumudu transform method to derive explicit solutions to Cauchy problem (1-2).

Theorem 2.1. The functions

$$
y_{i}(t)=t^{i} E_{\alpha, i+1}\left(\lambda t^{\alpha}\right), \quad i=0, \ldots, m-1
$$

yields the fundamental system of solutions to Cauchy problem (1-2).

Proof. Applying the Sumudu transform to (1), we have

$$
u^{-\alpha} \bar{y}(u)-\sum_{k=0}^{m-1} y^{(k)}(0) u^{\alpha-k}-\lambda \bar{y}(u)=0
$$

or

$$
\bar{y}(u)=\frac{b_{0} u^{-\alpha}+b_{1} u^{1-\alpha}+\cdots+b_{n-1} u^{n-\alpha-1}}{u^{-\alpha}-\lambda}=\sum_{k=0}^{m-1} \frac{b_{k} u^{k-\alpha}}{u^{\alpha-k}}
$$

where $\bar{y}(u)$ denotes $S[y(t)]$. Thus, from Lemma (2.1), we derive the following solution to the Eq.(1)

$$
y(x)=\sum_{r=0}^{m-1} b_{k} t^{k} E_{\alpha, i+1}\left(\lambda t^{\alpha}\right)
$$

It follows

$$
\left\{\begin{array}{c}
y_{l}^{(k)}(0)=0, \quad k, l=0, \ldots, m-1, \quad k \neq l \\
y_{l}^{(k)}(0)=1, \quad k=0, \ldots, m-1, \quad k=l .
\end{array}\right.
$$

By the above relations Wronskian $W(t)=\left[y_{l}^{(k)}(t)\right]_{l, k=0}^{m-1}$ at the point zero is equal 1: $W(0)=1 \$$. Thus the proof is complete.

Theorem 2.2. Fractional Diffusion-Wave equation

$$
\frac{\partial^{\alpha} U(x, t)}{\partial t^{\alpha}}=c^{2} \frac{\partial^{\beta} U(x, t)}{\partial x^{\alpha}}
$$

along with initial conditions 


$$
U(x, 0)=f_{1}(t), \quad \frac{\partial}{\partial t} U(x, 0)=f_{2}(t)
$$

where $f_{i}(t), i=1,2$, are continuous and $c$ is constant has its solution given by

$$
U(x, t)=\frac{1}{\sqrt{2 \pi}} \int_{-\infty}^{+\infty} e^{(-i k x)} E_{\alpha, 1}\left(-b t^{\alpha}\right) f_{1}^{*}(k) d k+\frac{1}{\sqrt{2 \pi}} \int_{-\infty}^{+\infty} t e^{(-i k x)} E_{\alpha, 1}\left(-b t^{\alpha}\right) f_{2}^{*}(k) d k
$$

where $f_{i}^{*}(k), i=1,2$, are the fourier transform of $f_{i}(t), i=1,2$.

Proof. Applying the Sumudu transform with respect to time variable $t$ and using initial condition (4) in (3), we find

$$
u^{-\alpha} \bar{U}(x, u)-u^{-\alpha} f_{1}(x)-u^{1-\alpha} f_{2}(x)=c^{2} \frac{\partial^{\beta} \bar{U}(x, u)}{\partial x^{\beta}} .
$$

Taking the fourier transform of the above equation, we get

$$
u^{-\alpha} \bar{U}^{*}(k, u)-u^{-\alpha} f_{1}{ }^{*}(k)-u^{1-\alpha} f_{2}{ }^{*}(k)=-c^{2}|k|^{\beta} \bar{U}^{*}(k, u) .
$$

Solving for $\bar{U}^{*}(k, u)$, we get

$$
\bar{U}^{*}(k, u)=\frac{u^{-\alpha} f_{1}{ }^{*}(k)}{u^{-\alpha}+c^{2}|k|^{\beta}}-\frac{u^{-\alpha} f_{2}{ }^{*}(k)}{u^{1-\alpha}+c^{2}|k|^{\beta}} .
$$

Inverting the sumudu transform with the help Lemma (2.1), we obtain

$$
\bar{U}^{*}(k, t)=E_{\alpha, 1}\left(-b t^{\alpha}\right) f_{1}{ }^{*}(k)+t E_{\alpha, 1}\left(-b t^{\alpha}\right) f_{2}{ }^{*}(k),
$$

where $b=-c^{2}|k|^{\beta}$. Finally, by taking fourier transform we get the required result of DiffusionWave equation in terms of generalized Mittag-Leffler function

$$
U(x, t)=\frac{1}{\sqrt{2 \pi}} \int_{-\infty}^{+\infty} e^{(-i k x)} E_{\alpha, 1}\left(-b t^{\alpha}\right) f_{1}^{*}(k) d k+\frac{1}{\sqrt{2 \pi}} \int_{-\infty}^{+\infty} t e^{(-i k x)} E_{\alpha, 1}\left(-b t^{\alpha}\right) f_{2}^{*}(k) d k
$$

\section{ACKNOWLEDGEMENTS}

The authors would like to thank the reviewers. The work is supported by the Islamic Azad Universities, Neka and Sari Branches.

\section{References}

[1] G.K. Watugala, Int. J. Math. Edu. Sci. Tech., 24 (1)p35 (1993).

[2] G.K. Watugala, Math. Eng. Industry., 6 (4)p319 (1998).

[3] G.K. Watugala, Math. Eng. Industry, 8 (4)p293 (2002).

[4] M.A. Asiru, Int. J. Math. Edu. Sci. Tech., 33 (3)p441 (2002).

[5] F.M. Belgacem, A.A. Karaballi and S.L. Kalla, Math. Prob. Eng., 3p103 (2003). 
[6] S. Weerakoon, Int. J. Math. Edu. Sci. Tech., 25 (2)p227 (1994).

[7] S. Weerakoon, Int. J. Math. Edu. Sci. Tech., 29 (4)p618 (1998).

[8] V.B. Chaurasia and J. Singh, Appl. Math. Sci., 57 (4)p2843 (2010).

[9] A. Kilicman, H. Eltayeb and R.P. Agarwal, Abs. Appl. Anal, Article ID: 598702, 11 pages, (2010).

[10] K.S. Miller, B. Ross, An Introduction to the Fractional Calculus and Fractional Differential Equations, Wiley, New York., 1993.

[11] I. Podlubny, Fractional differential equations, Academic Press, San Diego, CA, (1999).

[12] S.G. Samko, A.A. Kilbas, O.I. Marichev, Fractional Integrals and Derivatives, Theory and Applications, Gordon and Breach,Yverdon., 1993.

[13] R. Darzi, A. Neamaty, Y.Darzi, B. Mohammadzadeh, J. Math. Comput. Sci (TJMCS)., 4 (1)p102 (2012). 